\title{
Treatment of Multiple Sclerosis -
}

\section{Relationship between Vitamin $D$ and Interferon $\beta-1 b$}

\author{
Bruce Taylor, ${ }^{1}$ Harold Moses, ${ }^{2}$ Friedemann Paul, ${ }^{3}$ Gustavo Suarez ${ }^{4}$ and Mark Rametta ${ }^{5}$ \\ 1. Menzies Institute for Medical Research, University of Tasmania, Hobart, Australia; 2. Vanderbilt University, Nashville, Us; 3. Department of Neurology and NeuroCure \\ Clinical Research Center, Charité - Universitätsmedizin Berlin, Berlin, Germany; 4. Global Medical Affairs, Neurology, Bayer HealthCare Pharmaceuticals Inc., US; \\ 5. US Medical Affairs, Neurology, Bayer HealthCare Pharmaceuticals Inc., Whippany, New Jersey, us
}

\begin{abstract}
There are many reports suggesting an association between vitamin D status and both the development of multiple sclerosis (MS) and its course. This relationship and the effects of vitamin D and interferon $\beta$-1b (IFN $\beta$ - $1 \mathrm{~b}$ ) in the treatment of patients are reviewed in the BEtaferon/ Betaseron in Newly Emerging multiple sclerosis For Initial Treatment (BENEFIT) and the Betaferon/Betaseron Efficacy Yielding Outcomes of a New Dose in multiple sclerosis (BEYOND) studies. In the BENEFIT study the average serum 25-hydroxyvitamin D (25[OH]D) levels strongly predicted MS disease activity and progression. The probability of clinically definite MS (CDMS) and magnetic resonance imaging (MRI) activity was lower in these clinically isolated syndrome (CIS) patients with $25(\mathrm{OH}) \mathrm{D}$ levels $\geq 50 \mathrm{nmol} / \mathrm{L}$ and in those starting with IFN $\beta-1 \mathrm{~b}$. Furthermore, there was a beneficial effect on relapse rate, occurrence of new active MRI lesions and disease progression for a 50 nmol/L increase in 25(OH)D levels. Similarly, in relapsing-remitting (RR) MS patients from the BEYOND study serum 25(OH)D levels were inversely associated with MRI markers of MS activity. Genetic analysis of patients from these studies indicated that there may be a benefit in monitoring and managing vitamin D levels in early MS patients treated with IFN $\beta-1 \mathrm{~b}$ and a cumulative number of risk alleles predict lower $25(\mathrm{OH}) \mathrm{D}$ levels in $\mathrm{CIS}$ and RRMS patients. Further studies have suggested that some of the IFN $\beta$-1 $\mathrm{b}$ therapeutic effects on relapse could be mediated through modulation of vitamin D metabolism. Thus, there seems to be a benefit on clinical and MRI measures if patients are treated with both vitamin $D$ and IFN $\beta$ - $1 \mathrm{~b}$. There is a need to further evaluate this effect in clinical trials. The relationship between vitamin D and MS disease activity along with the effects of vitamin D and IFN $\beta$-1b in the treatment of MS patients is reviewed.
\end{abstract}

\section{Keywords}

Vitamin D, 25-hydroxyvitamin D, multiple sclerosis, interferon $\beta$-1b

\begin{abstract}
Disclosures: Bruce Taylor receives research support from the National Health and Medical Research Council (NHMRC) Australia, MS Research Australia and the Royal Hobart Hospital Research Foundation. He has served on advisory boards for Novartis Biogen and Genzyme and has received travel assistance from Novartis, Biogen and Teva. Harold Moses has served as a consultant to Biogen, Teva, Serono, Medimmune, Novartis, Genzyme, NIH (Neuronext) and Bayer. Friedemann Paul receives funding from Deutsche Forschungsgemeinschaft (EXc 257), Bundesministerium für Bildung und Forschung (BMBF Competence Network Multiple Sclerosis KKNMS), EU FP7 (combims.eu), Guthy Jackson Charitable Foundation, National Multiple Sclerosis Society of the USA, research support and personal compensation for activities with Alexion, Bayer, Biogen, MerckSerono, Chugai, MedImmune, Novartis, SanofiGenzyme and Teva. Gustavo Suarez is an employee of Bayer HealthCare Pharmaceuticals. Mark Rametta is an employee of Bayer HealthCare Pharmaceuticals.

Acknowledgement: Medical writing support was provided by Ray Ashton, Richmond Medical Communications, which was funded by Bayer HealthCare Pharmaceuticals Inc. All named authors meet the ICMJE criteria for authorship for this manuscript, take responsibility for the integrity of the work as a whole and have given final approval for the version to be published.

Open Access: This article is published under the Creative Commons Attribution Noncommercial License, which permits any non-commercial use, distribution, adaptation and reproduction provided the original author(s) and source are given appropriate credit.

Received: 29 September 2015 Accepted: 23 October 2015 Citation: European Neurological Review, 2015;10(2):124-30 http://doi.org/10.17925/ENR.2015.10.02.124 Correspondence: Bruce Taylor, Menzies Institute for Medical Research, University of Tasmania, Hobart, Australia. E: bruce.taylor@utas.edu.au.
\end{abstract}

Support: The publication of this article was supported by Bayer HealthCare Pharmaceuticals Inc. The views and opinions expressed in the article are those of the authors and not necessarily those of Bayer Healthcare Pharmaceuticals Inc.

There is increasing evidence that vitamin D status is associated with both the development of multiple sclerosis (MS) and also the modulation of disease activity and possibly progression. Several publications have reported that the risk of MS is higher in patients with low vitamin $D$ intake or serum 25-hydroxyvitamin D (25[OH]D) levels, for example due to low sun exposure. ${ }^{1,2}$ However, the findings of studies examining the relationship between vitamin D and MS disease activity are difficult to compare as the patients enrolled often had variable disease duration and it has been problematic to decide if low vitamin D levels were a consequence of MS activity (reverse causality) or if low levels early in the disease can predict long-term progression and disability.3,4
A recent publication analysed systematic reviews and meta-analyses to summarise the risk factors associated with MS. ${ }^{5}$ Of the 44 meta-analyses (416 primary studies), 11 had high significance $(p<0.001)$. Anti-EpsteinBarr nuclear antigen (anti-EBNA) immunoglobulin (Ig)-G seropositivity, infectious mononucleosis and smoking showed the strongest consistent evidence of an association in these high significance studies. Vitamin D status did not reach significance as a risk factor. However, it is important to note that vitamin D measured at the onset of MS may not necessarily reflect vitamin D levels at other significant times in a person's life, which may predispose them to later development of MS. Measure of prior sun exposure as potential, although not absolute, proxy for vitamin D levels 
in a Caucasian population have suggested that lifetime sun exposure, as well as sun exposure in the adolescent period, may be more important factors in MS risk than recent sun exposure. ${ }^{6,7}$ Effects of vitamin D levels on disease activity may be easier to assess as the exposure (vitamin D) is contemporaneous with the outcome (disease activity).

Here we review the relationship between vitamin D and MS disease activity and the effects of vitamin $D$ and interferon $\beta-1 b$ (IFN $\beta-1 b)$ in the treatment of MS in patients from the BEtaferon/Betaseron in Newly Emerging multiple sclerosis For Initial Treatment (BENEFIT) and the Betaferon/Betaseron Efficacy Yielding Outcomes of a New Dose in multiple sclerosis (BEYOND) studies. Furthermore, the molecular mechanisms underlying the impact of vitamin D on MS disease activity and the possible genetic component associated with vitamin D and MS are considered.

\section{Is Vitamin D Status an Early Predictor of Multiple Sclerosis Activity and Progression? Clinically Isolated Syndrome Patients from the BENEFIT Study}

The patients were assessed in order to determine if vitamin D status early in the MS disease process influenced the long-term disease course. ${ }^{8}$ BENEFIT was a randomised, placebo-controlled, double-blind trial that studied patients with a first event suggestive of MS treated with either IFN $\beta$-1b or placebo. Recruited patients were mostly white of European ancestry. The BENEFIT study was originally set up to compare early and delayed treatment with IFN $\beta$-1b. To fulfil the inclusion criteria, patients needed a minimum of two clinically silent magnetic resonance imaging (MRI) lesions. The placebo-randomised patients remained on placebo for 2 years or until a second clinical event occurred and a diagnosis of clinically definite MS (CDMS) could be made. All patients were eligible to enter a prospectively planned follow-up when they received open-label IFN $\beta$ - $1 \mathrm{~b}$ for up to 5 years after randomisation. Table 1 summarises selected characteristics of BENEFIT participants, the baseline characteristics were similar between patients with $25(\mathrm{OH}) \mathrm{D}$ levels $<50 \mathrm{nmol} / \mathrm{L}$ and $\geq 50 \mathrm{nmol} / \mathrm{L}$, except for the median number of T2 lesions, which were higher in the lower $25(\mathrm{OH}) \mathrm{D}$ group. Serum samples were collected at baseline, 6, 12 and 24 months for the measurement of $25(\mathrm{OH}) \mathrm{D}$ levels. Most statistical analyses were restricted to patients who had 25(OH)D measured at 6 and 12 months to reduce bias from seasonal variation, as the seasonally synchronous baseline, 12-month and 24-month samples could give a biased estimate of the year-round status of vitamin D. The clinical and MRI assessments of time to a definite diagnosis of MS, MS activity and MS progression were analysed. MS activity was assessed as the number of relapses and new active lesions on brain MRI, while percentage change in T2 lesion volume and the percentage change of brain volume represented MRI markers of progression. Clinically, progression was determined by the Expanded Disability Status Score (EDSS) score over time, with month 6 as the primary reference point. All analyses were adjusted for sex, age at baseline, treatment at randomisation, baseline T2 lesion score and type of clinically isolated syndrome (CIS) (monofocal versus multifocal).

Patients with higher 25(OH)D levels, were generally younger, with a lower BMI, smaller number of T2 lesions, smaller T2 lesion volume and higher brain volume at CIS. In total, $46.6 \%$ of patients converted to CDMS and this was strongly related to serum 25(OH)D.

In the first 12 months after CIS, the average serum 25(OH)D level strongly predicted MS activity and progression in the subsequent 4 years. The

\section{Table 1: Selected Characteristics at Baseline by Average 25(OH)D levels at 12 Months - BENEFIT Study}

\begin{tabular}{|c|c|c|}
\hline & $\begin{array}{l}25(\mathrm{OH}) \mathrm{D} \\
<50 \mathrm{nmol} / \mathrm{L}\end{array}$ & $\begin{array}{l}25(\mathrm{OH}) \mathrm{D} \\
\geq 50 \mathrm{nmol} / \mathrm{L}\end{array}$ \\
\hline Number of patients* & 251 & 213 \\
\hline 25(OH)D, median (range), nmol/L & $39(19-50)$ & $60(50-98)$ \\
\hline Age at recruitment, mean (SD), years & $31.3(7.6)$ & $30.1(7.2)$ \\
\hline Female, \% & 71.1 & 70.0 \\
\hline Randomised to IFN $\beta-1 \mathrm{~b}, \%$ & 59.8 & 65.3 \\
\hline Monofocal onset, \% & 52.6 & 52.1 \\
\hline Number of T2 lesions at baseline, median (Q1-Q3) & $20(8-38)$ & $15(7-37)$ \\
\hline
\end{tabular}

${ }^{*} n=464$ patients as one patient only had 25-hydroxyvitamin $D(25(\mathrm{OH}) \mathrm{D})$ measures at 24 months. The baseline characteristics were similar between patients with $25(\mathrm{OH}) \mathrm{D}$ levels $<50 \mathrm{nmol} / \mathrm{L}$ and $\geq 50 \mathrm{nmol} / \mathrm{L}$ except for the median number of T2 lesions, which were higher in the lower 25(OH)D group. BENEFIT = BEtaferon/Betaseron in Newly Emerging multiple sclerosis For Initial Treatment. Source: reproduced with permission from Ascherio et al., 2014. ${ }^{8}$

probability of CDMS was lower in the group of patients with levels of $25(\mathrm{OH}) \mathrm{D} \geq 50 \mathrm{nmol} / \mathrm{L}$ and in the subgroup of patients starting with IFN $\beta$ $1 \mathrm{~b}$ from randomisation compared with those with levels $<50 \mathrm{nmol} / \mathrm{L}$ ( $p=0.048$ and $p=0.0043$ after 1 year for all patients and IFN $\beta$ - $1 \mathrm{~b}$-treated patients, respectively). Furthermore, MRI activity, defined as the cumulative number of new active lesions (new or enlarging T2 lesions, new gadolinium-enhancing lesions), was significantly reduced in all patients and in the subgroup of patients treated with IFN $\beta-1 \mathrm{~b}$ from randomisation with $\geq 50 \mathrm{nmol} / \mathrm{L} 25(\mathrm{OH}) \mathrm{D}$ levels versus those with levels $<50 \mathrm{nmol} / \mathrm{L}$ ( $p=0.002 ; p=0.0055$, respectively) (see Figures 1 and 2). In addition, the percent change in $\mathrm{T} 2$ lesion volume and brain volume were strongly inversely associated with $25(\mathrm{OH}) \mathrm{D}$ levels $(\mathrm{p}=0.008$ and 0.005 , respectively) and the subgroup of patients starting with early treatment with IFN $\beta-1 b$ ( $p=0.016$ and 0.0037 , respectively). Furthermore, there was no difference in the number of patients with plasma $25(\mathrm{OH}) \mathrm{D}<50 \mathrm{nmol} / \mathrm{L}$ or $\geq 50 \mathrm{nmol} / \mathrm{L}$ reporting flu-like symptoms at 6 and 12 months. ${ }^{9,10}$

Patients were also assessed according to their 25(OH)D levels in increments of $50 \mathrm{nmol} / \mathrm{L}$. Overall, there was also a non-significant $27 \%$ decrease in relapse rate for a $50 \mathrm{nmol} / \mathrm{L}$ increment in $25(\mathrm{OH})$ D. This association was stronger among patients at both 6 and 12 months; in this group a significantly lower relapse rate with increasing serum 25(OH)D was observed after 12 months. The rate of occurrence of new active MRI lesions decreased with increasing serum 25(OH)D and a $50 \mathrm{nmol} / \mathrm{L}$ increase in the average 25(OH)D level significantly predicted a lower number of lesions. Measures of MS progression (change in brain volume, T2 lesion volume and EDSS score) were also inversely associated with increased $25(\mathrm{OH}) \mathrm{D}$ with each indicating a beneficial effect. For a $50 \mathrm{nmol} / \mathrm{L}$ increase in $25(\mathrm{OH}) \mathrm{D}$, the rate of brain volume loss was lower by $0.27 \%(p=0.12)$, T2 lesion volume decreased by $20 \%$ / year $(p<0.001)$ and average EDSS score reduced by 0.16 steps $(p=0.11)$.

Although associations were generally stronger for MRI than for clinical outcomes, the latter were still remarkable considering the overall low rate of relapses ( 0.2 per year) and small EDSS score change (median change, 0.0) in BENEFIT. The results emphasise the importance of identifying and correcting 25(OH)D insufficiency early in the course of MS and also indicate that early treatment with IFN $\beta-1 \mathrm{~b}$ has an additive effect with $25(\mathrm{OH}) \mathrm{D}$ to reduce disease severity and progression in both clinical and imaging outcomes without impacting tolerability to IFN $\beta-1 \mathrm{~b}$. 


\section{Figure 1: Levels of $25(\mathrm{OH})$ Vitamin $D \geq 50 \mathrm{nmol} / \mathrm{L}$ Significantly Reduced the Risk of CDMS in the BENEFIT Study}
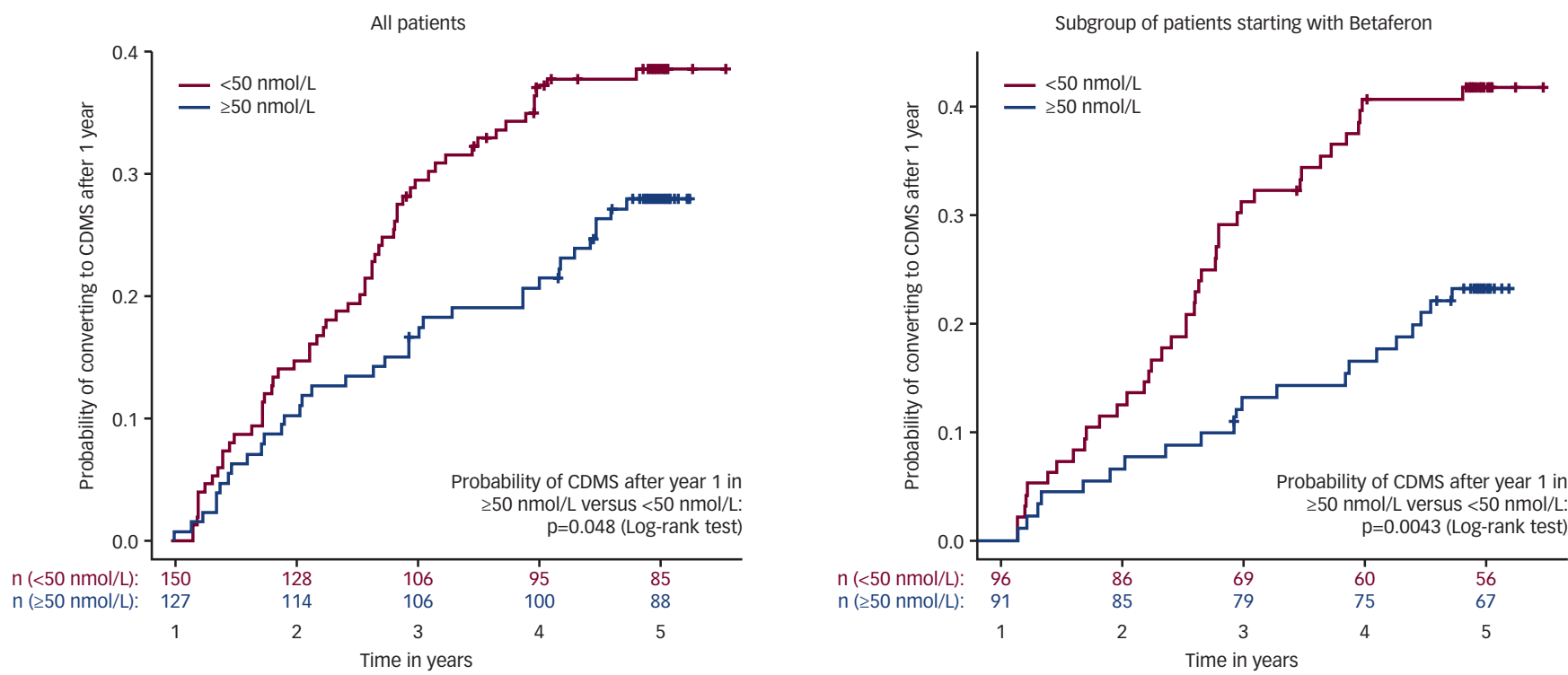

$25(\mathrm{OH}) \mathrm{D}=25$-hydroxyvitamin D; BENEFIT = BEtaferon/Betaseron in Newly Emerging multiple sclerosis For Initial Treatment; CDMS = clinically definite multiple sclerosis. Source: reproduced with permission from Ascherio et al., 2014. ${ }^{8}$

\section{Figure 2: Levels of $25(\mathrm{OH})$ Vitamin D $\geq 50 \mathrm{nmol} / \mathrm{L}$ Significantly Reduced Magnetic Resonance Imaging Activity in the BENEFIT Study}
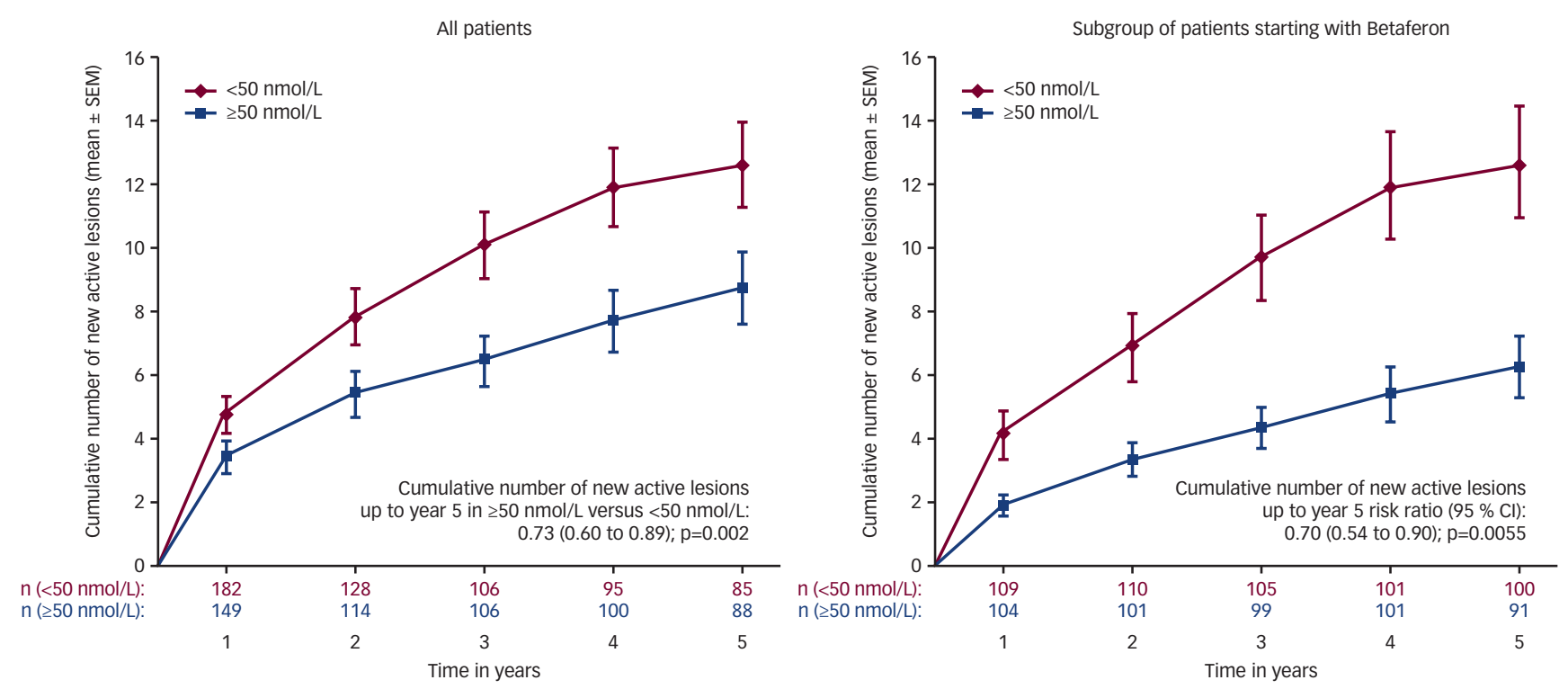

BENEFIT = BEtaferon/Betaseron in Newly Emerging multiple sclerosis For Initial Treatment; $\mathrm{Cl}=$ confidence interval; $\mathrm{MRI}=$ magnetic resonance imaging; $\mathrm{SEM}=\mathrm{standard}$ error of the mean. Source: reproduced with permission from Ascherio et al., 2014. ${ }^{8}$

\section{Relapsing-remitting Multiple Sclerosis Patients} from the BEYOND Study

The BEYOND study included patients from Western and Eastern Europe, North America and the Southern hemisphere who had been diagnosed with relapsing-remitting MS (RRMS) using the McDonald 2001 criteria and were treatment naïve. The regional subgroup baseline characteristics are summarised in Table 2.11 The three study arms were treatment with IFN $\beta$-1b (either $500 \mu \mathrm{g}$ or $250 \mu \mathrm{g}$ ) or glatiramer acetate (GA) $20 \mathrm{mg}$ and the mean time on study was 2.3 years. ${ }^{11}$ Blood samples from the two IFN $\beta-1 \mathrm{~b}$ arms were analysed to determine the impact of averaged vitamin D levels on MS disease course. The GA group was not included in the analysis. In total, 1,482 patients had at least two 25(OH)D measurements 6 months apart. For
25(OH)D status, the primary exposure variable was the cumulative average of season-adjusted 25(OH)D values up to 12 months with the adjustment performed within each region. Season adjustment was completed as described previously. ${ }^{2}$ The $50 \mathrm{nmol} / \mathrm{L}$ increments and also the $<37.5,37.5-49.9,50-<74.9,75-99.9$ and $\geq 100 \mathrm{nmol} / \mathrm{L}$ were compared.

The multivariate statistical analysis was adjusted for age, gender, disease duration, region of residence, baseline EDSS and treatment dose. Crosssectional analysis compared season-adjusted $25(\mathrm{OH}) \mathrm{D}$ versus baseline MRI and versus 12-month MRI. Finally, Iongitudinal analysis compared the season-adjusted 25(OH)D levels in first year with clinical/MRI disease course during the study. 
Table 2: Selected Characteristics of Participants (250 and $500 \mu \mathrm{g}$ ) by Region of Recruitment BEYOND Study

\begin{tabular}{|c|c|c|c|c|c|c|c|c|c|c|}
\hline & Argentina & Australia & Brazil & $\begin{array}{l}\text { Western } \\
\text { Europe }\end{array}$ & Mediterranean & Scandinavia & Russia & $\begin{array}{l}\text { Eastern } \\
\text { Europe }\end{array}$ & $\begin{array}{l}\text { North } \\
\text { America }\end{array}$ & $\mathrm{p}$ Value \\
\hline Number & 49 & 28 & 62 & 220 & 164 & 29 & 180 & 295 & 429 & \\
\hline \multicolumn{11}{|c|}{ Season-adjusted 25(OH)D, nmol/L } \\
\hline Mean (SD) & $54.2(19.5)$ & $67.4(22.3)$ & $72.1(20.5)$ & $58.2(24.7)$ & $61.8(24.5)$ & $62.7(21.8)$ & $43.4(14.0)$ & $52.5(19.0)$ & $65.4(30.1)$ & $<0.0001$ \\
\hline Median (IQR) & $54.6(28.9)$ & $66.4(18.8)$ & $68.9(3.7)$ & $53.5(39.7)$ & $58.2(32.6)$ & $54.1(28.4)$ & $41.9(20.0)$ & $50.1(22.4)$ & $59.7(35.8)$ & \\
\hline$\%$ male & 41 & 14 & 24 & 34 & 35 & 24 & 28 & 32 & 26 & 0.05 \\
\hline Age, mean (SD), years & $37.2(9.5)$ & $36.0(9.0)$ & $32.9(9.0)$ & $35.7(9.4)$ & $35.8(9.0)$ & $26.7(7.1)$ & $35.9(9.7)$ & $32.2(82)$ & $38.7(9.0)$ & $<0.0001$ \\
\hline $\begin{array}{l}\text { Age at onset, } \\
\text { mean (SD), years }\end{array}$ & $30.6(8.5)$ & $31.0(8.9)$ & $29.9(9.2)$ & $31.4(9.3)$ & $31.1(8.9)$ & $31.4(7.3)$ & $29.3(9.3)$ & $27.7(7.8)$ & $34.4(9.1)$ & $<0.0001$ \\
\hline $\mathrm{BMI}\left(\mathrm{kg} / \mathrm{m}^{2}\right)$, mean $(\mathrm{SD})$ & $25.1(4.6)$ & $25.5(4.8)$ & $25.1(4.7)$ & $25.4(5.0)$ & $24.4(4.4)$ & $23.9(3.4)$ & $23.3(4.0)$ & $23.1(3.9)$ & $28.3(6.8)$ & $<0.0001$ \\
\hline \multicolumn{11}{|c|}{ Baseline Disease Characteristics } \\
\hline EDSS (SD) & $2.9(1.4)$ & $2.4(1.0)$ & $2.5(1.2)$ & $2.0(1.0)$ & $2.0(1.1)$ & $1.9(1.2)$ & $3.1(1.1)$ & $2.5(1.1)$ & $2.3(1.1)$ & $<0.0001$ \\
\hline $\begin{array}{l}\text { Number of relapses in } \\
\text { past year (at baseline), } \\
\text { mean (SD) }\end{array}$ & $2.0(1.0)$ & $2.3(0.9)$ & $2.4(1.1)$ & $1.8(0.9)$ & $1.9(0.9)$ & $1.9(0.7)$ & $2.4(1.1)$ & $2.1(1.01)$ & $1.9(0.9)$ & 0.0002 \\
\hline $\begin{array}{l}\text { Disease duration, } \\
\text { median (IQR) }\end{array}$ & $7.1(6.2)$ & $5.6(6.5)$ & $3.6(3.3)$ & $4.8(5.0)$ & $5.2(5.6)$ & $5.8(6.1)$ & $7.2(6.6)$ & $5.1(5.1)$ & $4.8(6.2)$ & $<0.0001$ \\
\hline MSSS, mean (SD) & $4.8(2.7)$ & $4.8(2.4)$ & $5.4(2.6)$ & $3.9(2.3)$ & $3.8(2.4)$ & $3.6(2.4)$ & $5.2(2.2)$ & $4.9(2.1)$ & $4.8(2.5)$ & $<0.0001$ \\
\hline $\begin{array}{l}\text { Volume of T2 } \\
\text { hyper-intense } \\
\text { lesions, mean (SD), } \mathrm{cm}^{3}\end{array}$ & $13.4(12.9)$ & $7.5(9.2)$ & $9.0(8.7)$ & $7.4(7.9)$ & $7.7(7.9$ & $7.5(8.5)$ & $13.2(13.4)$ & $11.7(12.2)$ & $6.7(8.8)$ & $<0.0001$ \\
\hline $\begin{array}{l}\text { Volume of } \mathrm{T} 1 \\
\text { non-enhancing } \mathrm{T} 1 \\
\text { hypointense lesions, } \\
\text { mean (SD), } \mathrm{cm}^{3}\end{array}$ & $2.8(3.2)$ & $1.7(2.8)$ & $1.3(2.6)$ & $1.3(2.1)$ & $1.4(2.1)$ & $1.5(2.3)$ & $3.1(5.2)$ & $2.2(3.6)$ & $1.1(2.7)$ & $<0.0001$ \\
\hline $\begin{array}{l}\text { T1-enhancing lesions, } \\
\text { median (IQR) }\end{array}$ & $4(5)$ & $1.9(3)$ & $3.4(8.0)$ & $2.0(1)$ & $1.8(2)$ & $1.3(1)$ & $3.1(7.0)$ & $3.0(3)$ & $1.8(4.0)$ & 0.0005 \\
\hline $\begin{array}{l}\text { Normalised brain } \\
\text { volume, mean (SD), } \mathrm{cm}^{3}\end{array}$ & 1,452 (130) & 1,529 (84) & $1,517(98.9)$ & $1,501(110)$ & $1,475(100)$ & $1,500(106)$ & $1,468(114)$ & $1,473(114)$ & 1,503 (108) & $<0.0001$ \\
\hline
\end{tabular}

$25(\mathrm{OH})=25$-hydroxyvitamin; BEYOND = Betaferon/Betaseron Efficacy Yielding Outcomes of a New Dose in multiple sclerosis; $B M I=$ body mass index; EDSS= Expanded Disability Status Score; IQR = interquartile range; MSSS = Multiple Sclerosis Severity Score; SD = standard deviation. Source: Reproduced with permission from Fitzgerald et al., 2015.

The 25(OH)D levels were lowest in Eastern Europe and highest in Australia, Brazil and North America - as expected levels reflected the seasonal variation in sunlight for each region. Significant differences were also observed in several clinical parameters. Younger age, higher volume of T2 hyperintense lesions and longer disease duration were seen in Eastern Europe and Russia compared with Western Europe and North America. In the cross-sectional analyses adjusted for age, sex, treatment group (IFN $\beta$-1b 250 versus $500 \mu g$ ), baseline EDSS score, disease duration, region of residence, baseline $25(\mathrm{OH})$ D levels, reported as estimates (95\% confidence interval [CI]) were inversely associated with T2 lesion volume with a $50 \mathrm{nmol} / \mathrm{L}$ higher level corresponding to a difference of $-0.11 \mathrm{~cm}^{3}$ ( $\log \mathrm{T} 2$ lesion volume) -0.20 to $-0.02 ; p=0.02$, and the total number of T1-enhancing lesions $-0.24,-0.45$ to $-0.02 ; p=0.03$ at baseline. The association between 25(OH)D and MRI parameters at 12 months gave similar results, with a difference in $\log \mathrm{T} 2$ lesion volume of $-0.19 \mathrm{~cm}^{3},-0.31$ to 0.07 ; $\mathrm{p}=0.003$. The difference at 12 months for log $\mathrm{T} 1$ hypointense lesion volume was $-0.07 \mathrm{~cm}^{3},-0.14$ to $0.00 ; p=0.055$ and during year 1 the rate of $\mathrm{T} 2$ lesion count $0.78,0.62$ to $0.99 ; \mathrm{p}=0.04$. Furthermore, $25(\mathrm{OH})$ $\mathrm{D}$ levels were also inversely associated with the cumulative number of new active lesions (the sum of new T2 lesions and T1-enhancing lesions) between baseline and 12 months ( $0.76,0.60$ to $0.98 ; p=0.03)$. In longitudinal analysis, the relative rate (RR) of cumulative new active lesions was reduced by $31 \%$ (RR 0.69, 95\% Cl, 0.55-0.86; $\mathrm{p}=0.001$ ) for each $50 \mathrm{nmol} / \mathrm{L}$ increase in 25(OH)D level in all patients. This inverse association was also strong and significant in analyses restricted to patients with 25(OH)D levels greater than $50 \mathrm{nmol} / \mathrm{L}$ (RR 0.62; $95 \%$
$\mathrm{Cl}, 0.46-0.84 ; \mathrm{p}=0.002)$, and was consistently observed in each of the four regions (see Figure 3).

In the cross-sectional analysis between baseline 25(OH)D levels and the number of relapses in the previous 2 years, no significant association was observed. However, baseline $25(\mathrm{OH}) \mathrm{D}$ level was inversely associated with EDSS scores at baseline with a $50 \mathrm{nmol} / \mathrm{L}$ higher level of 25(OH)D corresponding to a mean difference of 0.28 in EDSS scores (95\% $\mathrm{Cl},-0.40$ to $-0.16 ; p<0.001$ ).

In summary, in these RRMS patients, serum 25(OH)D levels were inversely associated with MRI markers of MS activity and there was a similar magnitude across geographic regions despite differences in average levels. Furthermore, the association was similar in different patient subgroups according to demographic or clinical characteristics. The associations between 25(OH)D and relapses or clinical progression were not significant, which may be due to the low relapse rate as well as a relatively short follow-up.

Another study investigated whether higher levels of 25(OH)D were protective in patients treated with GA compared with IFN $\beta$ (Comprehensive Longitudinal Investigation of Multiple Sclerosis at the Brigham and Women's Hospital, Partners MS Center [CLIMB] study). ${ }^{12}$ Levels of 25(OH)D were determined in 247 RRMS patients treated with either GA or IFN $\beta$ as their first disease-modifying drug (DMD). Higher 25(OH)D significantly predicted longer time to first event (clinical and MRI endpoint) in the IFN group ( $p=0.013)$, but not with $G A(p=0.38)$. 


\section{Figure 3: Relative Rate of Cumulative New Active Lesions versus Average Baseline, 6- and 12-month 25(OH)D Level Stratification by Geographic Region - BEYOND Study}

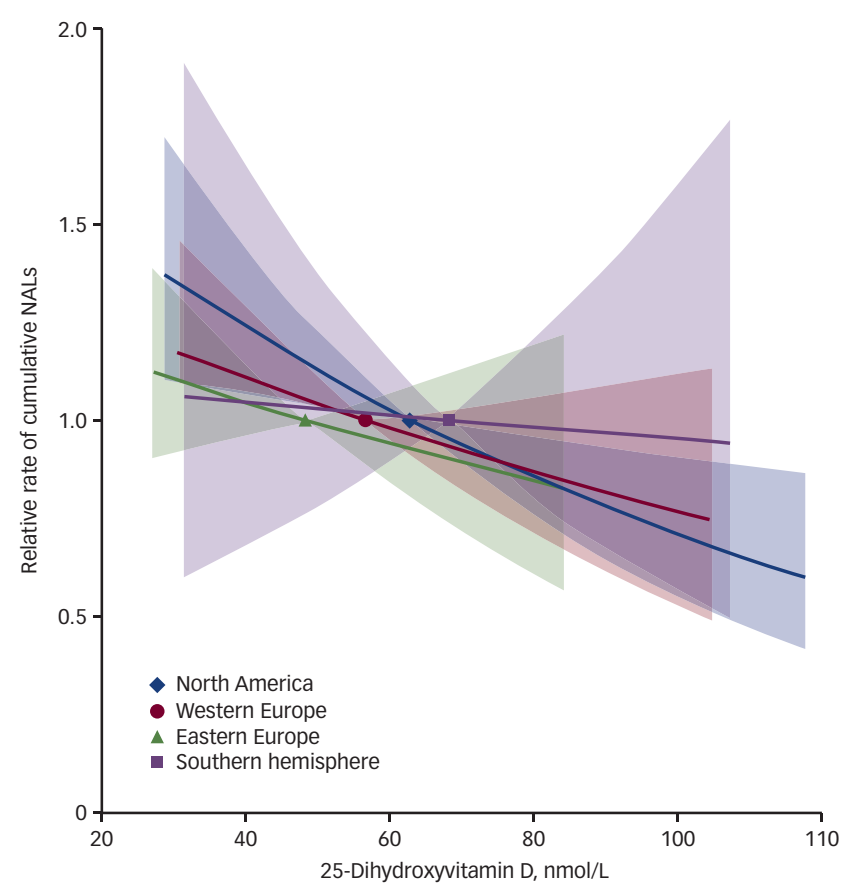

The solid lines and shaded regions represent the relative rate ratios of cumulative new active lesions (NALS). NALs for changes in 25-hydroxyvitamin D $(25(\mathrm{OH}) \mathrm{D})$ relative to the median level and the corresponding $95 \%$ confidence intervals, respectively. Analyses were adjusted for age, sex, randomisation status, baseline Expanded Disability Status scale score and disease duration (1, 2-5, 6-10 and >10 years). Models assume a linear association between the logarithm of the rate of cumulative NALS and serum 25(OH)D. Analyses using cubic splines revealed no significant deviation from linearity. To convert 25(OH)D values to nanograms per millilitre, divide by 2.496. BEYOND = Betaferon Betaseron Efficacy Yielding Outcomes of a New Dose in multiple sclerosis. Source: Reproduced with permission from Fitzgerald et al., 2015.11

\section{Molecular Mechanisms Underlying the Impact of Vitamin D on Multiple Sclerosis Disease Activity}

A global gene expression analysis of the patients from BENEFIT showed that $25(\mathrm{OH}) \mathrm{D}$ regulates the expression dynamics of a large gene-gene interaction system, which primarily regulates immune modulatory processes modulating MS activity. ${ }^{13} \mathrm{~A}$ decrease in MS activity was observed with increasing 25(OH)D levels. A number of known targets of IFN $\beta-1 \mathrm{~b}$ and a regulator of sphingosine-1-phosphate bioavailability were identified in the complex network of genes regulated by $25(\mathrm{OH})$ D. These results indicate a possible benefit in early MS patients treated with IFN $\beta-1 b$, of monitoring and managing vitamin D levels.

\section{Genetic Modification of 25(OH)D Levels in Multiple Sclerosis}

Candidate single nucleotide polymorphisms (SNPS) were analysed by genome-wide association studies (GWAS) to assess their impact on 25(OH)D levels in IFN $\beta$-1b-treated CIS and RRMS patients from the BENEFIT and BEYOND trials $(n=815) .^{14}$

All assessed candidate SNPS in DHCR7 (7-dehydrocholesterol reductase), GC (vitamin D binding protein) and CYP2R1 (cytochrome P450, family 2, subfamily $R$, polypeptide 1 ) genes were significantly associated with 25(OH)D levels $(\mathrm{p} \leq 0.05)$. Furthermore, a 25(OH)D risk score to indicate the odds ratio of being $25(\mathrm{OH}) \mathrm{D}$ deficient was derived from the cumulative count of risk alleles per patient. As the number of risk alleles increased, levels of 25(OH)D decreased (see Figure 4). The findings indicated that the effects of multiple risk alleles are additive and may be used to develop a 25(OH)D genetic risk score in MS patients (higher score represents a higher possibility of low 25(OH) D). This vitamin D risk score could increase awareness of the risk of deficiency in MS patients in the absence of 25(OH)D measurement. The results indicated that the cumulative number of risk alleles predict lower 25(OH)D levels in CIS/RRMS patients.

\section{Vitamin $D$ and Interferon $\beta$}

Studies in animal models of MS, randomised clinical trials and cohort studies have assessed whether there is an association between vitamin $\mathrm{D}$ and IFN $\beta$. The 1,25[OH][2]D[3] analogue, TX527 (19-nor14,20-bisepi-23-yne-1-1,25[OH][2]D[3]) has diminished calcemic effects but enhanced anti-proliferative and pro-differentiating properties on normal and malignant cell types in vivo compared with $1,25[\mathrm{OH}][2] \mathrm{D}[3] .^{15}$ Studies in an experimental autoimmune encephalomyelitis (EAE) animal model demonstrated that TX527 attenuates EAE. ${ }^{16}$ Further studies evaluated combinations of TX527, IFN $\beta$ and cyclosporine $A$ in this model and showed that the combination of TX527 and IFN $\beta$ was significantly more effective in ameliorating the disease than either treatment alone. In addition, there was no effect with IFN $\beta$ and cyclosporine A but when TX527 was added to this combination virtually complete protection occurred. The authors suggested that the effect of IFN $\beta$ and TX527 should be evaluated in a clinical trial of MS. ${ }^{16}$

The safety and efficacy of adding vitamin $\mathrm{D}_{3}$ to IFN $\beta$ - $1 \mathrm{~b}$ treatment was assessed in a double-blind, placebo-controlled, randomised trial in 66 MS patients over 1 year. ${ }^{17}$ In treated patients, the median change in T2 burden of disease was reduced in treated patients $\left(83 \mathrm{~mm}^{3}\right.$ versus $287 \mathrm{~mm}^{3}$ in the placebo group). Levels of $25(\mathrm{OH}) \mathrm{D}$ in serum increased with $84 \%$ of patients achieving $>85 \mathrm{nmol} / \mathrm{L}$ in the IFN $\beta-1 \mathrm{~b}$ group compared with placebo $(p<0.0001)$. In addition, there was a trend towards a reduction in disability accumulation and improved timed tandem walk in the treatment group with no significant difference in annual relapse rate or adverse events. ${ }^{17}$

In a prospective cohort comprising 178 patients with clinically definite MS (CDMS), Stewart et al. measured 25(OH)D levels in serum twice a year together with a direct patient review and a questionnaire regarding sun exposure and relapse information in order to determine if IFN $\beta$ treatment was associated with vitamin D levels and if they interact with respect to relapse rate. ${ }^{18}$ Patients treated with IFN $\beta$ had significantly higher mean $25(\mathrm{OH}) \mathrm{D}$ levels than non-treated patients $(p<0.001)$. This was mediated by an interaction between a patient's sun exposure and IFN $\beta$ therapy, with treated patients achieving approximately three times the amount of 25(OH)D for each hour of sun exposure than those not on therapy. Furthermore, there was an association between $25(\mathrm{OH}) \mathrm{D}$ and reduced relapse risk only in treated patients $(p<0.001)$. The reduction in relapse rate was only seen in IFN $\beta$-treated patients with higher 25(OH)D levels whereas in those with insufficiency the relapse rate increased. These findings suggest that some of the therapeutic effects of IFN $\beta$ on relapse may be mediated through modulation of vitamin D metabolism. It could therefore be beneficial to monitor vitamin D status and supplement if necessary in patients treated with $\operatorname{IFN} \beta .{ }^{18}$

Reder et al. investigated whether vitamin D enhanced IFN $\beta$ signalling by studying in vitro intracellular IFN $\beta$ response to combinations of vitamin 
$D$ and IFN $\beta$ in treatment-naïve RRMS and progressive MS patients and those receiving IFN $\beta$ or GA. ${ }^{19}$ Vitamin D enhanced IFN $\beta$ activation of STAT1 by $30-70 \%$ in untreated patients and by $30-150 \%$ in those treated with IFN $\beta$, but with only modest effects with GA. This increase in IFN signaling may provide a mechanism for the benefits of vitamin $D$ in preventing and ameliorating MS, both before and during IFN $\beta$ $1 \mathrm{~b}$ treatment. Vitamin $\mathrm{D}$ may also help overcome the effects of low intrinsic serum IFN- $\alpha$ and $\beta$ levels in MS. ${ }^{20}$

A study of RRMS patients treated with IFN $\beta$ and given vitamin D at either 800 or 4,370 IU/day assessed for the post-injection cytokine surge that is associated with flu-like symptoms. ${ }^{21}$ In the low-dose group, serum IL17 increased significantly $(p=0.037$ ) but the response was inconsistent in the high-dose group with $40 \%$ having decreased IL-17 levels and $45 \%$ increased levels. No significant changes occurred in the serum concentration of IL-10 and IFN- $\alpha$. These results showed no benefit of vitamin D supplementation on flu-like symptoms but illustrate its immunomodulatory properties.

\section{Conclusion}

Vitamin D status was assessed in patients who participated in the BENEFIT and BEYOND studies in order to explore the relationship between serum vitamin $\mathrm{D}$ level and the disease course. In the BENEFIT patients, serum 25(OH)D level predicted MS activity and progression. There appeared to be a correlation with serum $25(\mathrm{OH})$ D levels $\geq 50 \mathrm{nmol} / \mathrm{L}$ and a positive benefit. The findings indicate the potential benefit of identifying patients with vitamin D insufficiency and correcting it. Furthermore, results from the BENEFIT cohort suggest that vitamin $D$ and IFN $\beta$ - $1 \mathrm{~b}$ have an additive effect on disease activity.

Similarly in the BEYOND study cohort, serum 25(OH)D levels had an inverse association with MRI markers of MS disease activity, which was similar in those from different locations and with various demographic and clinical characteristics. There was a non-significant association with relapses possibly as a result of the low relapse rate and relatively short follow-up period.

Preliminary results in EAE suggest that vitamin D has a synergistic effect with IFN $\beta$. Furthermore IFN $\beta$ has an additive effect with $25(\mathrm{OH}) \mathrm{D}$ on changes in gene expression known to reduce gadolinium-enhancing lesion counts, which suggests that improving vitamin D levels in such MS patients may be beneficial. 25(OH)D also shows systemic gene expression regulation on 25(OH)D target gene sets. Moreover, GWAS studies have identified candidate SNPS that were significantly associated with 25(OH)D levels.

Many studies of vitamin D and MS have caveats, which potentially cast doubts on their validity. There is a general consensus that betterdesigned trials are needed to establish more robust evidence - especially multicentre, randomised, controlled, clinical trials using clinical, immunological and MRI outcomes. To address this problem Efficacy of Vitamin D Supplementation in Multiple Sclerosis (EVIDIMS), a German, multicentre, stratified, randomised, controlled, double-blind, phase II trial was initiated.22 This trial aimed to randomise $80 \mathrm{CIS}$ or RRMS patients treated with IFN $\beta$ - 1 b to 20,400 or $400 \mathrm{IU} 25(\mathrm{OH}) \mathrm{D}$ every other day for 18 months. The primary endpoint is the cumulative number of new T2 lesions on cranial MRI during the treatment period. Secondary endpoints include other MRI parameters, such as progression of brain atrophy over the study period and number and volume of T1-hypointense and T2-hyperintense lesions. As retinal thinning is increasingly recognised

\section{Figure 4: Concentration by Number of Risk Alleles - BENEFIT and BEYOND Studies}

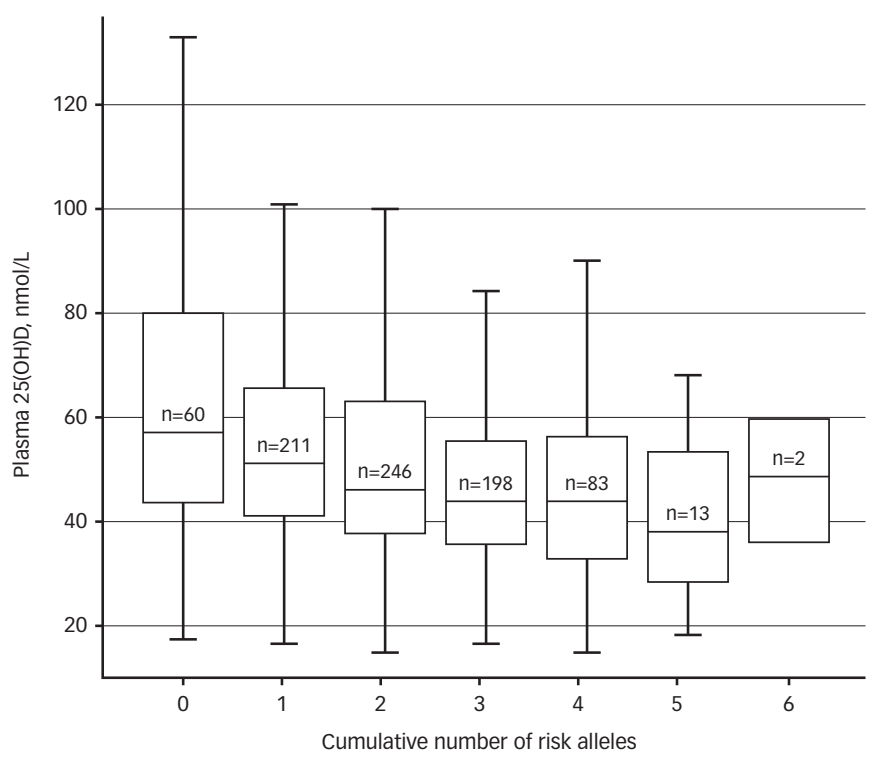

$25(\mathrm{OH}) \mathrm{D}=25$-hydroxyvitamin D; BENEFIT = BEtaferon/Betaseron in Newly Emerging multiple sclerosis For Initial Treatment; BEYOND = Betaferon/Betaseron Efficacy Yielding Outcomes of a New Dose in multiple sclerosis.Source: Reproduced with permission from Munger et al., 2014.14

as a promising surrogate marker of tissue loss and neurodenegeration in MS, retinal nerve fibre layer thickness and macular volume measured by optical coherence tomography are additional secondary outcome parameters. ${ }^{23-25}$ The study has completed recruitment, however, results have not been published to date. Another such trial is the Supplementation of VigantOL ${ }^{\circledR}$ Oil (Merck KGaA, Darmstadt, Germany) versus placebo as add-on in patients with RRMS receiving Rebif ${ }^{\circledR}$ (Merck Serono S.A. Geneva, Switzerland) treatment (SOLAR) study. ${ }^{26}$ This 96-week, three-arm, multicentre, double-blind, randomised, placebo-controlled phase II trial will assess vitamin $D_{3}(7,000 \mathrm{IU}$ daily for 4 weeks then 14,000 daily for a further 92 weeks) as add-on therapy to subcutaneous IFN $\beta$-1a $44 \mu \mathrm{g}$ three times weekly in RRMS patients. The study aimed to randomise 348 patients with RRMS and 25(OH)D serum levels $<150 \mathrm{nmol} / \mathrm{l}$ in a 1:1 ratio to high-dose vitamin $D_{3}$ versus placebo. A composite of clinical and MRI parameters was chosen as primary endpoint. The primary MRI endpoint is the mean number of combined unique active lesions at week 48 and the primary clinical endpoint the proportion of relapse-free patients at week $96 .{ }^{26}$ Several additional clinical and MRI endpoints will be assessed. This study has been completed, however, data have not yet been presented. The investigators anticipate that these studies will provide additional insights into the effect of vitamin D on IFN treatment. There is a lack of consensus concerning the definition of vitamin D deficiency and sufficiency both in the general population and in disorders such as MS. Until the results of the EVIDIMS and SOLAR studies are available, it would seem prudent that people with MS, regardless of their treatment status, have sufficient levels of vitamin $D$, according to local guidelines. There is growing evidence that this should be at least $50 \mathrm{nmol} / \mathrm{L}$ for those on IFN $\beta$.

It seems likely that there is an association between vitamin D levels and IFN $\beta$ treatment in MS patients with an additive benefit for the combination. ${ }^{27}$ Many studies have identified benefits on clinical and MRI measures in treating patients with both therapies. Ongoing clinical trials may provide more definitive evidence for this potentially beneficial combination. 


\section{Multiple Sclerosis}

Whether there may be a beneficial effect of a combination therapy of vitamin $D_{3}$ with $G A$ is under investigation in the Vitamin $D$ to Ameliorate Multiple Sclerosis (VIDAMS) trial (NCT01490502). ${ }^{28}$ One hundred and seventy-two patients with RRMS are randomised in a 1:1 ratio to 5,000 IU or 600 IU oral vitamin $D_{3}$ add on to GA over 96 weeks. The primary endpoint is the proportion of patients with a relapse at 2 years. Secondary and tertiary endpoints include additional clinical and MRI parameters. The study had not yet completed enrolment in spring $2015 .^{29}$
1. Munger $\mathrm{KL}$, Zhang $\mathrm{SM}$, $\mathrm{O}$ 'Reilly $\mathrm{E}$, et al., Vitamin $\mathrm{D}$ and incidence of multiple sclerosis, Neurol, 2004;62:60-5.

2. Munger KL, Levin LI, Hollis BW, et al., Serum 25-hydroxyvitamin D level and risk of multiple sclerosis, JAMA, 2006;296:2832-8.

3. Ascherio A, Marrie RA, Vitamin D in MS: a vitamin for 4 seasons, Neurol, 2012;79:208-10.

4. Dörr J, Döring A, Paul F, Can we prevent or treat multiple sclerosis by individualised vitamin D supply?, EPMA J, 2013;4:4.

5. Belbasis L. Bellou V, Evangelou E, et al., Environmental risk factors and multiple sclerosis: an umbrella review of systematic reviews and meta-analyses, Lancet Neurol, 2015;14:263-73

6. Van der Mei IA, Ponsonby AL, Dwyer T, et al., Past exposure to sun, skin phenotype, and risk of multiple sclerosis: casecontrol study, BMJ, 2003;327:316.

7. Lucas RM, Ponsonby AL, Dear K, et al., Sun exposure and vitamin $\mathrm{D}$ are independent risk factors for CNS demyelination, Neurology, 2011:76:540-8.

8. Ascherio A, Munger KL, White R, et al., Vitamin $\mathrm{D}$ as an early predictor of multiple sclerosis activity and progression, JAMA Neurol, 2014;71:306-14.

9. Ascherio A, Munger KL, White R, et al., Presented at: the Annual Meeting of the American Academy of Neurology, Philadelpia, PA, USA; 26 April-3 May 2014.

10. Ascherio A, Fitzgerald K, Munger KL, et al., Vitamin D as a predictor of multiple sclerosis activity and progression in patients with multiple sclerosis on interferon beta-1b. parion Presentation FC4.4.Presented at: ECTRIMS, Boston, USA
12 September 2014. of vitamin D levels with multiple sclerosis activity and progression in patients receiving interferon beta-1b, JAMA
Neurol, 2015; Epub ahead of print.

2. Rotstein D, Healy B, Malike MT, et al., Differential effects of vitamin D in GA-versus IFN-treated MS patients, Neurol, 2014;82:S24.005

13. Munger KL, Köchert K, Simon KC, et al., Molecular mechanism underlying the impact of vitamin D on disease activity in MS Annals Clin Transl Neurol, 2014;72:605-17.

14. Munger KL, Köchert K, Fitzgerald K, et al., Genetic modification of 25(OH)D levels in MS. Poster P411, Presented at: ECTRIMS, Boston, USA: 10-13 September 2014

15. González-Pardo V, Verstuyf $A$, Boland $R$, Russo de Boland $A$, Vitamin D analogue TX 527 down-regulates the NF-Kb pathway and controls the proliferation of endothelial cells transformed by Kaposi sarcoma herpesvirus, British $J$ Pharmacol, 2013;169:1635-45

16. van Etten E, Gysemans C, Branisteanu DD, et al., Novel insights in the immune function of the vitamin D system: synergism with interferon-beta, J Steroid Biochem Mol Biol, 2007;103:546-51.

17. Soilu-Hänninen M. Aivo J Lindström BM et al., A randomised, double-blind, placebo controlled trial with vitamin D3 as an add on treatment to interferon $\beta$ - $1 \mathrm{~b}$ in patients with multiple sclerosis, JNNP, 2012:83:565-71.

18. Stewart N, Simpson S, van der Mei I, et al., Interferon- $\beta$ and serum 25-hydroxyvitamin D interact to modulate relapse risk in MS, Neurol, 2012;79:254-60

19. Reder AT, Wang Z, Causevic $S$, et al., Vitamin D enhances in vitro IFN- $\beta$ responses in MS. Poster P.533. Presented at: ECTRIMS, Barcelona, Spain 7-10 October 2015.

20. Feng $X$, Petraglia $\mathrm{AL}$, Chen $\mathrm{M}$, et al., Low expression of interferon-stimulated genes in active multiple sclerosis is linked to subnormal phosphorylation of STAT1 I Neuroimmunol, 2002;129:205-15.

21. Golan D, Halhal B, Glass-Marmor L, et al., Vitamin D supplementation for patients with multiple sclerosis treated with interferon-beta: a randomized controlled trial assessing the effect on flu-like symptoms and immunomodulatory properties, BMC Neurol, 2013;13:60

22. Dorr J, Ohlraun S, Skarabis H, Friedemann P, Efficacy of vitamin D supplementation in multiple sclerosis (EVIDIMS trial): study protocol for a randomized controlled trial, Trials, 2012;13:15.

23. Brandt AU, Oberwahrenbrock $\mathrm{T}$, Ringelstein $\mathrm{M}$, et al., Primary retinal pathology in multiple sclerosis as detected by optical coherence tomography, Brain, 2011;134:1-3.

24. Pfueller $C F$, Brandt AU, Schubert F, et al., Metabolic changes in the visual cortex are linked to retinal nerve fiber layer thinning in multiple sclerosis, PLoS One, 2011;6:e18019.

25. Bock M, Brandt AU, Kuchenbecker J, et al., Impairment of contrast visual acuity as a functional correlate of retinal fibre layer thinning and total macular volume reduction in multiple sclerosis, Br J Ophthalmol, 2012;96:62-7.

26. Smolders J, Hupperts R, Barkof F, et al., Efficacy of vitamin $\mathrm{D}_{3}$ as add-on therapy in patients with relapsing-remitting multiple sclerosis receiving subcutaneous interferon beta-1a: A Phase II, multicenter, double-blind, randomized, placebocontrolled trial, J Neurol Sci, 2011;311:44-9.

27. Bowling $A$, Integrating vitamin $D$ into overall therapeutic strategy for MS, Int I MS Care, 2015;17:17-21.

28. Bhargava P, Cassard S, Steele SU, et al., The vitamin D to ameliorate multiple sclerosis (VIDAMS) trial: study desig for a multicenter, randomized, double-blind controlled trial of vitamin D in multiple sclerosis, Contemp Clin Trials, 2014;39:288-93.

29. Vitamin D Supplementation in Multiple Sclerosis. Available at: https://clinicaltrials.gov/ct2/show/NCT01490502?term $=M$ ULTIPLE+SCLEROSIS\&intr=vitamin+d\&rank=11 (accessed 20 October 2015). 